\title{
Harms Associated with Usage of Senna Leaves (Sana Makki) in COVID-19
}

\author{
Irshad Hussain ${ }^{1, *}$, Che Suraya Zin², Ehsanullah Malik², Muhammad Shahzad Raza4 \\ ${ }^{1}$ Institute of Pharmacy, SMBB Medical University Larkana, Pakistan \\ ${ }^{2}$ Kulliyyah of Pharmacy, International Islamic University Malaysia \\ ${ }^{3}$ Department of Surgery, SMBB Medical University Larkana,Pakistan \\ ${ }^{4}$ NMC, University of Health Sciences Lahore, Pakistan
}

\section{Dear Editor,}

A stimulant laxative; Senna is an ornamental plant of the family Fabaceae. There has been ancient use of the drug for its laxative/purgative action. The plant is located in tropics. It contains the constituents anthraquinones, dianthrones and anthrones. Sennosides A\& B are derivatives of anthraquinones at $2.5 \%$ minimal concentration. Leaves of the plants have been used for laxative/purgative action by pediatricians, pediatric surgeons and pediatric gastroenterologists [1]. Senna is described as a safe option to treat constipation. There have been some media reports of the misuse of Senna in Pakistan for COVID-19 infection. Governor of the Sindh Province has mentioned the use of Senna (Sana Makki) in COVID-19 patients [2]. A YouTube video has been viral of a person, a UK resident advising the use of leaves of Senna to cure the COVID-19 and very unscientifically presenting himself for penalty if his claims are proved false provided that the drug is used as prescribed by his method. While his method was not based on any clinically published trial of him or others. Illiterate peoples have started using the leaves as suggested in the video. Impressed from convincing technique of the person, there are heaps of recipes of Sana Makki leaves mentioned in different links of Facebook related with the methods told by Dr Nazeer in his video. Due to usage by community in COVID19 , the price of Senna leaves has jumped from Rs 250 per kg (USD 1.5) to around Rs 2500 per Kg (USD 15). Medical professionals have described such news for of using Senna in COVID-19 as unproven, baseless and misleading and warned the public not to use the herb to avoid unintended side effects that outweigh some of its health benefits [3]. Word "Senna" was combined with the word "COVID-19" or "SARS" using "AND" as boolean and was searched at
Pubmed advanced database. There was none retrieval of clinical evidence regarding the use and effectiveness of Senna in the COVID-19 infection. Only two articles were retrieved from the searched results which were unrelated to the clinical use of Senna. There has been no availability of any peer reviewed or published clinical description regarding the use of senna in the SARS, MERS and COVID-19. Senna has been approved by FDA for its use in constipation/laxative action and is included among the Over-the-counter (OTCs) drugs requiring no prescription [4]. Gastrointestinal symptoms have been reported due to COVID-19 and have been included in the symptoms of the disease [5]. Use of laxatives in GIT symptoms can definitely synergize the motility leading to diarrheal symptoms and dehydration affecting the electrolyte balance of the patients. Loss of nutrients in diarrhea and vomiting can also reduce the immunity of patients reducing the powerful natural defense of the body versus the COVID-19. It has been recommended by the American Herbal Plant Associations (AHPA) regarding the labelling instructions of the senna leave products to not use them if having diarrhea or abdominal pain and requiring the consultation of health care provider for use in pregnant and nursing mothers as the constituents of Senna irritate the bowl linings for laxative action [4]. There have been use of Senna infusions in chronic constipation as similar use recommendations in the prophetic medicines. Medicinal plants have common anti-inflammatory properties in providing symptomatic relief in common cold, fever and sore throat. The myth of Senna use in COVID-19 has been derived from such basis ignoring the laxative/purgative action of the drug. However safer herbal options like green tea and honey can be soothing in COVID-19 symptoms. Use of Senna in 
COVID-19 patients has been strongly discouraged due to its enormous side effects like diarrhea, water loss, electrolyte imbalance and hypokalemia. Such side effects can be harsh for those patients already in critical conditions. There have been many reported interactions of Senna with many drugs and it is not suited for heart, liver and kidney patients. Thereby, the use of Senna remains incalculable and the risks can outweigh the benefits. Senna can also not be used for long time due to possibility of its dependency on laxatives [6]. WHO has encouraged the clinical investigations to find safe and effective herbs in the treatment of COVID-19 [7]. Senna leaves may not be used for the prophylaxis or treatment of COVID-19 without having any sufficient clinical data as the drug is potent laxative and can raise the complications of COVID-19 patients instead of benefits by augmenting diarrhea and other gastrointestinal problems.

\section{REFERENCES}

1. Vilanova-Sanchez A, Gasior AC, Toocheck N, et al. Are Senna based laxatives safe when used as long term treatment for constipation in children?. J Pediatr Surg. 2018; 53(4):722-7. doi:10.1016/j.jpedsurg.2018.01.002.

2. https://www.dawn.com/news/1557883.

3. https://tribune.com.pk/story/2234073/1-tribunefact-check-sana-makki-cure-COVID-19/.

4. https://www.webmd.com/vitamins/ai/ingredientmon o-652/senna.

5. Lijing Yang, and Lei Tu. Implications of gastrointestinal manifestations of COVID-19. Lancet gastroenterology and Hepatology. 2020, 12.

https://www.thelancet.com/action/showPdf?pii=S2 468-1253\%2820\%2930132-1.

6. https://www.youtube.com/watch? $\mathrm{v}=\mathrm{N} \times 1 \mathrm{~K} 1 \times 4 \mathrm{~N} 9 \mathrm{ok}$ (A Report by BBC Urdu).

7. https://www.afro.who.int/news/who-supportsscientifically-proven-traditionalmedicine?gclid=Cj0KCQjwiYL3BRDVARIsAF9E4G dN9SZEFe2Ez8_qIFBvF63sireJnUu1QbJsgMX27 FeQdZdk7GBbgRgaAtOvEALw_wcB 